Short Communication

\title{
Identification of a novel mutation at the primary dimer interface of GyrA conferring fluoroquinolone resistance in Clostridium difficile
}

\author{
Micheál Mac Aogáin a,*, Shauna Kilkenny ${ }^{\mathrm{a}}$, Claire Walsh ${ }^{\mathrm{a}}$, Sinéad Lindsay ${ }^{\mathrm{a}}$, \\ Geraldine Moloney ${ }^{\mathrm{a}}$, Trefor Morris ${ }^{\mathrm{b}}$, Sophie Jones ${ }^{\mathrm{b}}$, Thomas R. Rogers ${ }^{\mathrm{a}}$ \\ ${ }^{a}$ Department of Clinical Microbiology, Sir Patrick Dun Translational Research Laboratory, School of Medicine, Trinity College Dublin, Ireland \\ ${ }^{\mathrm{b}}$ Anaerobe Reference Laboratory, Public Health Wales, University Hospital of Wales, Cardiff, UK
}

\section{A R T I C L E I N F O}

\section{Article history:}

Received 3 June 2015

Accepted 28 September 2015

Keywords:

Clostridium difficile

DNA gyrase

Fluoroquinolone resistance

Genomics

\begin{abstract}
A B S T R A C T
The aim of this study was to determine whether alternative resistance mechanisms, other than mutation in the quinolone resistance-determining region (QRDR) of DNA gyrase, could confer fluoroquinolone resistance in Clostridium difficile. An in vitro-generated $C$. difficile mutant exhibiting increased fluoroquinolone resistance was isolated through antibiotic selection on ciprofloxacin. The QRDR of this mutant was investigated by chain-termination sequencing and was found to be devoid of mutation. To determine the nature of the non-QRDR resistance mechanism in this strain, the genomes of the mutant and wild-type strains were sequenced. The gyrBA region from a collection of clinical isolates exhibiting variable fluoroquinolone resistance levels was also sequenced and was compared with that present in 918 publicly available $C$. difficile genomic data sets. Whole-genome sequence analysis of the fluoroquinolone-resistant mutant revealed a single non-synonymous substitution (Ala384Asp) at the predicted primary dimer interface of GyrA, far beyond the classically defined QRDR. This novel mutation caused increased resistance to ciprofloxacin, ofloxacin, levofloxacin and moxifloxacin while conferring hypersusceptibility to novobiocin. Several novel extra-QRDR polymorphisms in $C$. difficile DNA gyrase were identified among clinical isolates, whilst observed fluoroquinolone resistance in strains devoid of gyrBA mutations confirmed the existence of DNA gyrase-independent resistance mechanisms in this species. In conclusion, we report the first non-QRDR mutation to confer fluoroquinolone resistance in $C$. difficile. Although the Ala384Asp substitution was not detected in clinical isolates, this study revealed a diversity of alternative extra-QRDR polymorphisms in DNA gyrase whose association with fluoroquinolone resistance warrants further investigation.
\end{abstract}

(C) 2015 International Society for Chemotherapy of Infection and Cancer. Published by Elsevier Ltd. All rights reserved.

\section{Introduction}

Clostridium difficile is a major intestinal pathogen with a disease spectrum ranging from mild diarrhoea to life-threatening pseudomembranous colitis [1]. An established risk factor for C. difficile infection (CDI) is prior antibiotic exposure, which causes reduced microbial diversity in the gut and the consequent loss of colonisation resistance [2]. Antibiotic resistance is a common characteristic of endemic $C$. difficile strains. Outbreaks in the 1990s are attributed to the emergence and international

\footnotetext{
* Corresponding author at: Department of Clinical Microbiology, Trinity College Dublin, Room 1.26, Sir Patrick Dun Translational Research Laboratory, St James's Hospital, Dublin 8, Ireland. Tel.: +353 18962876.

E-mail address: m.macaogain@tcd.ie (M. Mac Aogáin).
}

spread of virulent clindamycin-resistant strains, whilst globally disseminated strains of the 027/BI/NAP1 lineage exhibit resistance to the fluoroquinolones $[1,3]$.

Fluoroquinolone resistance is caused by mutations in DNA gyrase or topoisomerase IV that abrogate drug-target binding [4]. In C. difficile, resistance is linked to a homoplasic Thr82lle substitution in the quinolone resistance-determining region (QRDR) of the GyrA DNA gyrase subunit, a hallmark of globally disseminated 027/BI/NAP1 strains [5]. Several additional mutations that map to the QRDR of gyrA and gyrB have also been reported in C. difficile, whereas the type IV topoisomerase is absent in C. difficile as well as several other pathogenic species [6]. Molecular characterisation of fluoroquinolone resistance in C. difficile also suggests the existence of undetermined mechanisms among resistant strains lacking QRDR mutations [7,8]. Whilst the roles of alternative resistance mechanisms such as extra-QRDR 
mutations have been speculated upon, direct evidence for their involvement in fluoroquinolone resistance is lacking. We employed a forward genetic approach to reveal a novel mutation causing fluoroquinolone resistance in $C$. difficile and investigated its presence among clinical strains.

\section{Materials and methods}

\subsection{Bacterial strains used in this study}

Strains used in this study were isolated at St James's Hospital (Dublin, Ireland) between 2010 and 2011 from cases of CDI as part of routine diagnostic testing. Toxin production was confirmed in all strains with the Premier ${ }^{\mathrm{TM}}$ Toxin A and B Enzyme Immunoassay (Meridian Bioscience Inc., Cincinnati, $\mathrm{OH}$ ). Strains were subjected to PCR-based ribotyping performed at the Anaerobe Reference Laboratory (Cardiff, UK) as previously described [9].

\subsection{In vitro antibiotic selection conditions}

Between $10^{8}$ and $10^{9}$ cells of cultured clinical isolate SJH_18 [ciprofloxacin minimum inhibitory concentration (MIC) $=8 \mathrm{mg}$ / L] were plated onto pre-reduced brain-heart infusion agar supplemented with $0.5 \%$ yeast extract, $0.1 \%$ cysteine, $0.1 \%$ taurocholate and $32 \mathrm{mg} / \mathrm{L}$ ciprofloxacin (Sigma-Aldrich, Dublin, Ireland). Plates were incubated at $37^{\circ} \mathrm{C}$ under anaerobic conditions $\left(10 \% \mathrm{H}_{2}, 10 \% \mathrm{CO}_{2}\right.$ and $\left.80 \% \mathrm{~N}_{2}\right)$ until resistant colonies emerged (48-72 h).

\subsection{Antibiotic sensitivity testing}

Sensitivity to antibiotics was investigated using Etest strips (bioMérieux, Basingstoke, UK). Strains were assayed for sensitivity to ciprofloxacin, moxifloxacin, levofloxacin and ofloxacin as previously described, adopting the previous definition of 'highly resistant' (MIC $\geq 32 \mathrm{mg} / \mathrm{L}$ ) for ciprofloxacin and moxifloxacin in the absence of clinical breakpoints in $C$. difficile [8]. The relative levels of novobiocin sensitivity between strains were compared using antibiotic disks ( $5 \mu \mathrm{g} /$ disc; Oxoid Ltd., Basingstoke, UK).

\subsection{PCR amplification and sequencing of the QRDR and gyrBA open} reading frames (ORFs)

DNA was extracted from $C$. difficile using a High-Pure PCR Template Preparation Kit (Roche Diagnostics, West Sussex, UK). The QRDRs of gyrB (nucleotides 1059-1448) and gyrA (nucleotides 71-460) were amplified by PCR as described previously [6]. To investigate the entire gyrBA region, beyond the defined $\mathrm{QRDR}$, primers flanking the entire ORFs of gyrB and gyrA were designed. Resultant amplicons were purified using QIAquick PCR Clean Up and Gel Extraction Kits (QIAGEN, West Sussex, UK) and were sequenced externally (Source Biosciences, Dublin, Ireland). Primers used for amplification and sequencing reactions are listed in Supplementary Table S1.

\subsection{Whole-genome sequencing}

Sequencing of $C$. difficile genomic DNA was performed on an Illumina MiSeq platform at the TrinSeq sequencing facility (Trinity College Dublin, Ireland). This generated ca. 500,000,150-bp pairedend reads per strain. Short-read data obtained for $C$. difficile SJH_18 and SJH_18R have been deposited in the European Nucleotide Archive (ENA) under study accession no. PRJEB5003.

\subsection{Analysis of whole-genome short-read sequence data}

Sequencing reads from SJH_18 were assembled de novo using the NSilico Simplicity ${ }^{\mathrm{TM}}$ pipeline (Simplicity ${ }^{\mathrm{TM}}$ v.1.2) [10]. This generated an assembly for $\mathrm{SJH}_{-} 18$ against which reads from SJH_18R were mapped with Burrows-Wheeler short-read aligner [11]. The SAMtools analysis suite was used to detect single nucleotide variants (SNVs) present in SJH_18R [12]. Publicly available short-read data from 918 C. difficile strains was retrieved from the ENA to investigate the prevalence of non-synonymous SNVs in DNA gyrase among the broader C. difficile population. Data from 918 strains (Supplementary Table S2) was mapped to $C$. difficile 630 (AM180355.1) and variants in the gyrBA ORFs were called for each sequenced isolate. Non-synonymous SNVs in gyrB and gyrA were annotated with SNPdat [13].

\section{Results}

\subsection{Forward genetic identification of an extra-QRDR mutation conferring fluoroquinolone resistance in $\mathrm{C}$. difficile}

Following in vitro antibiotic selection on ciprofloxacin, the stable phenotypic mutant SJH_18R (ciprofloxacin MIC $\geq 32 \mathrm{mg} / \mathrm{L}$ ) was derived from strain $\mathrm{SJH}_{-} 18$ (ciprofloxacin $\mathrm{MIC}=8 \mathrm{mg} / \mathrm{L}$ ). In addition to ciprofloxacin, the $\mathrm{SJH} \_$18R mutant exhibited increased resistance to other fluoroquinolones including ofloxacin, levofloxacin and moxifloxacin while displaying concomitant hypersusceptibility to the aminocoumarin novobiocin (Table 1). As chain-termination sequencing revealed the QRDRs from the mutant and wild-type to be identical, the presence of a QRDRindependent mutation in $\mathrm{SJH}_{-}$18R was suspected. Whole-genome sequencing of SJH_18 and SJH_18R identified a single SNV difference between strains causing an Ala384Asp substitution in GyrA in SJH_18R (Table 1). This extra-QRDR mutation was thus identified as the cause of the pleotropic antibiotic resistance phenotype observed in SJH_18R.

\subsection{C. difficile clinical isolates exhibit QRDR-independent ciprofloxacin resistance}

To investigate QRDR-independent fluoroquinolone resistance in clinical isolates, we sequenced and compared the QRDRs of gyrB and gyrA from 31 additional isolates exhibiting variable fluoroquinolone resistance phenotypes (Table 2). Comparator strains SJH_18 and SJH_26 exhibited relatively lower ciprofloxacin MICs and were devoid of QRDR mutations, in line with their susceptibility profiles

Table 1

Antibiotic resistance profile of SJH_18 and SJH_18R.

\begin{tabular}{|c|c|c|c|c|c|c|c|}
\hline \multirow[t]{2}{*}{ Strain } & \multirow[t]{2}{*}{ Ribotype } & \multicolumn{5}{|c|}{ Antibiotic MIC (mg/L) } & \multirow[t]{2}{*}{ Non-synonymous DNA gyrase mutations } \\
\hline & & CIP & OFX & LEV & MXF & $\mathrm{NOV}^{\mathrm{a}}$ & \\
\hline SJH_18 & Type 110 & 8 & 8 & 4 & 0.5 & $7.3 \pm 1$ & $-{ }^{\mathrm{b}}$ \\
\hline SJH_18R & Type 110 & $\geq 32$ & $\geq 32$ & 8 & 1 & $13.7 \pm 1$ & GyrA, A384D \\
\hline
\end{tabular}

MIC, minimum inhibitory concentration; CIP, ciprofloxacin; OFX, ofloxacin; LEV, levofloxacin; MXF, moxifloxacin; NOV, novobiocin.

a Expressed as zone diameter around antibiotic disc $(\mathrm{mm})$.

b - Indicates no observed change relative to Clostridium difficile 630 (AM180355.1). 
Table 2

Comparison of fluoroquinolone minimum inhibitory concentrations (MICs) and non-synonymous mutations in DNA gyrase among Clostridium difficile clinical isolates.

\begin{tabular}{|c|c|c|c|c|c|c|c|}
\hline \multirow[t]{2}{*}{ Strain } & \multirow[t]{2}{*}{ Ribotype } & \multicolumn{2}{|c|}{ Antibiotic MIC (mg/L) } & \multicolumn{2}{|l|}{ QRDR } & \multicolumn{2}{|c|}{ Extra-QRDR ${ }^{\text {a }}$} \\
\hline & & CIP & MXF & GyrA & GyrB & GyrA & GyrB \\
\hline SJH_18 & Type 110 & 8 & 0.5 & $-{ }^{\mathrm{b}}$ & - & - & - \\
\hline SJH_26 & Type 010 & 8 & 1 & - & - & - & - \\
\hline SJH_5 & Type 020 & $\geq 32$ & 1 & - & - & - & I139R \\
\hline SJH_10 & Type 020 & $\geq 32$ & 1 & - & - & - & I139R \\
\hline SJH_27 & Type 020 & $\geq 32$ & 1 & - & - & - & I139R \\
\hline SJH_33 & Type 020 & $\geq 32$ & 1 & - & - & - & I139R \\
\hline SJH_43 & Type 020 & $\geq 32$ & 1 & - & - & - & I139R \\
\hline SJH_50 & Type 020 & $\geq 32$ & 1 & - & - & - & I139R \\
\hline SJH_24 & Type 014 & $\geq 32$ & 1 & - & - & - & I139R \\
\hline SJH_48 & Type 020 & $\geq 32$ & 1 & - & - & - & V130I \\
\hline SJH_14 & Type 011 & $\geq 32$ & 1 & - & - & - & V130I \\
\hline SJH_20 & Type 015 & $\geq 32$ & 1 & - & - & L345I & V130I \\
\hline SJH_17 & Type 081 & $\geq 32$ & 1 & - & - & - & - \\
\hline SJH_23 & Type 254 & $\geq 32$ & 1 & - & - & - & - \\
\hline SJH_41 & Type 001 & $\geq 32$ & 1 & - & - & - & - \\
\hline SJH_31 & Type 003 & $\geq 32$ & 1 & - & - & - & - \\
\hline SJH_69 & Type 002 & $\geq 32$ & 1 & - & - & - & - \\
\hline SJH_28 & Type 017 & $\geq 32$ & 1 & - & S366A & na ${ }^{c}$ & na \\
\hline \multirow[t]{2}{*}{ SJH_16 } & Type 078 & $\geq 32$ & 8 & A118T & S366V & na & na \\
\hline & & & & - & S416A & na & na \\
\hline SJH_34 & Type 087 & $\geq 32$ & 16 & - & D426N & na & na \\
\hline SJH_1 & Type 027 & $\geq 32$ & $\geq 32$ & T82I & - & na & na \\
\hline SJH_3 & Type 027 & $\geq 32$ & $\geq 32$ & T82I & S464T & na & na \\
\hline SJH_4 & Type 027 & $\geq 32$ & $\geq 32$ & T82I & S464T & na & na \\
\hline SJH_32 & Type 027 & $\geq 32$ & $\geq 32$ & T82I & S464T & na & na \\
\hline SJH_51 & Type 027 & $\geq 32$ & $\geq 32$ & T82I & S464T & na & na \\
\hline SJH_61 & Type 027 & $\geq 32$ & $\geq 32$ & T82I & S464T & na & na \\
\hline SJH_64 & Type 027 & $\geq 32$ & $\geq 32$ & T82I & S464T & na & na \\
\hline SJH_25 & Type 078 & $\geq 32$ & $\geq 32$ & T82I & - & na & na \\
\hline SJH_45 & Type 078 & $\geq 32$ & $\geq 32$ & T82I & - & na & na \\
\hline SJH_47 & Type 078 & $\geq 32$ & $\geq 32$ & T82I & - & na & na \\
\hline SJH_57 & Type 078 & $\geq 32$ & $\geq 32$ & T82I & - & na & na \\
\hline SJH_58 & Type 078 & $\geq 32$ & $\geq 32$ & T82I & - & na & na \\
\hline
\end{tabular}

CIP, ciprofloxacin; MXF, moxifloxacin; QRDR, quinolone resistance-determining region.

a Polymorphisms occurring outside the QRDR of GyrA (nt position 25-153) or GyrB (nt position 353-483).

b - Indicates no observed change relative to Clostridium difficile 630 (AM180355.1).

c na, not assayed (the wider gyrA and gyrB ORFs of strains in which QRDR mutations were detected were not further investigated).

(Table 2). In contrast, 15 strains that also lacked mutations in the QRDRs of either gyrA or gyrB none the less exhibited ciprofloxacin MICs of $\geq 32 \mathrm{mg} / \mathrm{L}$ (Table 2 ). Thus, in $50 \%$ of highly resistant strains (15/30), the observed ciprofloxacin MIC of $\geq 32 \mathrm{mg} / \mathrm{L}$ was not attributable to QRDR mutations. This suggested the presence of QRDR-independent resistance mechanisms. In addition, ribotyping combined with sequence analysis of gyrBA QRDR regions revealed high-level co-resistance to both ciprofloxacin and moxifloxacin (MIC $\geq 32 \mathrm{mg} / \mathrm{L}$ ) in 027 and 078 strains harbouring the classical Thr82Ile substitution in GyrA (Table 2). Other non-synonymous changes in the QRDR were also observed in strains belonging to ribotypes 087 (GyrB, Asp426Asn), 078 (GyrA, Ala118Thr; GyrB, Ser366Val, Ser416Ala) and 017 (GyrB, Ser366Val). In addition to the Thr82Ile mutation, six ribotype 027 strains also harboured a Ser464Thr substitution in GyrB (Table 2).

\subsection{Characterisation of non-synonymous extra-QRDR polymorphisms in ciprofloxacin-resistant C. difficile isolates}

To further investigate the mechanism of ciprofloxacin resistance in the 15 strains devoid of QRDR mutations, extended sequence analysis of the entire gyrB and gyrA ORFs was performed. This led to the identification of non-synonymous polymorphisms, located far beyond the characterised QRDRs of gyrB and gyrA, in ten isolates (Table 2). Five strains (SJH_25, SJH_45, SJH_47, SJH_57 and SJH_58) were devoid of mutation across the entire gyrBA ORF regions confirming the existence of additional, DNA gyraseindependent fluoroquinolone resistance mechanisms in $C$. difficile. Among strains where extra-QRDR mutations were identified, substitutions in GyrB (Ile139Arg and Val130Ile) represented the most frequent non-synonymous changes observed, whilst an extra-QRDR mutation in gyrA (Leu345Ile) was observed in a single strain (Table 2).

\subsection{Extra-QRDR DNA gyrase polymorphisms are present among divergent $\mathrm{C}$. difficile lineages}

Analysis of the gyrBA region in publicly available $C$. difficile genomic data confirmed GyrB substitutions Ile139Arg and Val130Ile to be prevalent among the broader $C$. difficile population (Fig. 1). Publicly available genomic data from 918 strains were analysed, of which 644 harboured at least one non-synonymous polymorphisms in the gyrBA region including substitutions occurring outside the QRDR, which were identified in 457 strains. The most prevalent extra-QRDR polymorphisms included Val130Ile, Ile139Arg and Gln160His in GyrB and Leu406Ile, Lys413Asn and Asp468Asn in GyrA, whilst known QRDR mutations including Thr82Ile were also observed (Fig. 1). Although the induced GyrA mutation Ala384Asp was not detected (other than in the in vitro-generated SJH_18R mutant), other polymorphisms including Leu406Ile and Lys413Asn were present at the predicted primary dimer interface region of GyrA (residues 386-426). Phylogenetic analysis confirmed the presence of extra-QRDR mutations among divergent multilocus sequence typing (MLST) types suggesting homoplasy (Fig. 1). However, mutations involving more dramatic side chain substitutions (i.e. GyrB, Ile139Arg; 


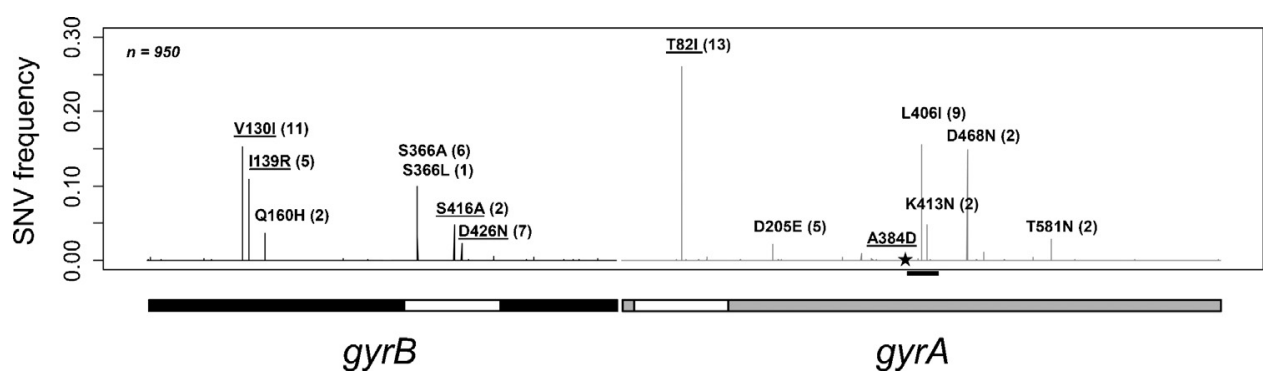

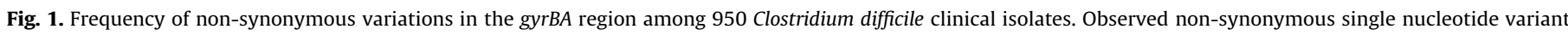

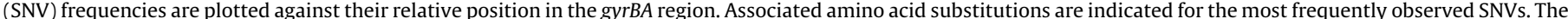

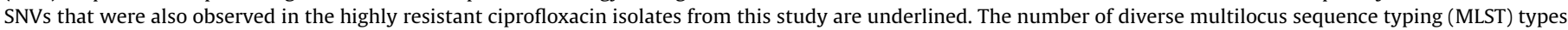

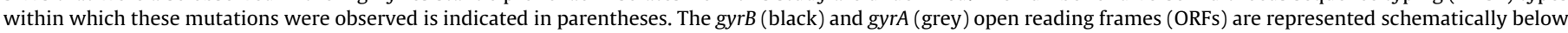

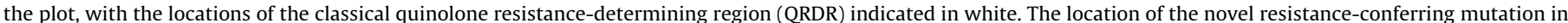
gyrA (Ala384Asp) identified in this study is indicated by a black star, and an adjacent black bar underlines the predicted GyrA primary dimer interface region.

GyrA, Asp205Glu, Asp468Asn) were generally restricted to related MLST lineages (Fig. 1; Supplementary Fig. S1; Supplementary Table S2). In addition to Leu406Ile and Lys413Asn, polymorphisms Gly401Arg, Gly401Val and Gln418Lys were observed at lower prevalence within the GyrA primary dimer interface (Supplementary Table S3).

\section{Discussion}

We report the first confirmed non-QRDR mutation conferring fluoroquinolone resistance in $C$. difficile. The Ala384Asp substitution identified in $\mathrm{SJH} \_$18R is located next to the predicted dimer interface region of GyrA, 231 amino acids outside the classical QRDR. In a previous study investigating fluoroquinolone resistance in Salmonella enterica, Blanc-Potard et al. characterised a pleiotropic mutation in gyrB causing ciprofloxacin resistance and concomitant novobiocin sensitivity. They subsequently identified an epistatic and compensatory mutation in gyrA that restored novobiocin sensitivity to wild-type levels while further augmenting fluoroquinolone resistance [14]. Notably, the epistatic mutation (Thr467Ser) was located at the dimer interface region, thus confirming the ability of mutation at this region in GyrA to influence both ciprofloxacin and novobiocin sensitivity, a finding consistent with the current observations in $C$. difficile. We speculate that the Ala384Asp mutation in $C$. difficile may be functionally analogous to the Thr467Ser mutation in Salmonella GyrA that results in a predicted destabilisation of the primary interface favouring a quinolone-resistant DNA gyrase conformation [14].

Having confirmed the ability of an extra-QRDR mutation to modulate resistance in vitro, we investigated whether such mutations were associated with resistance in $C$. difficile clinical isolates. DNA gyrase mutations occurring outside the QRDR are rarely investigated and their prevalence among fluoroquinoloneresistant strains is largely unknown $[7,8,15]$. Non-synonymous changes present outside the QRDR, including polymorphisms in GyrB (Ile139Arg and Val130Ile) and GyrA (Leu345Ile), were identified among our clinical strains exhibiting elevated ciprofloxacin MICs. In total, one-half (15/30) of the isolates exhibiting increased ciprofloxacin resistance were devoid of QRDR mutations, including ten isolates that harboured non-synonymous, extraQRDR mutations. Five strains harboured wild-type DNA gyrase alleles, providing the first demonstration that fluoroquinolone resistance can occur in $C$. difficile independent of DNA gyrase mutations. Whilst the precise mechanism of resistance in these strains remains to be determined, these findings add credence to the role of alternative fluoroquinolone resistance mechanisms such as drug efflux or reduced permeability in $C$. difficile, as has been suggested in other studies [7,8].
As in our clinical isolates, the Ala384Asp mutation was not detected among publicly available $C$. difficile genomic data sets, suggesting that it is uncommon. This may be due to its impact on novobiocin susceptibility or to other potentially deleterious effects on strain fitness associated with this DNA gyrase mutation. In contrast, analysis of deposited genomic data revealed a diversity of other extra-QRDR polymorphisms in DNA gyrase including the Val130Ile and Ile139Arg substitutions in GyrB that were also observed in the ciprofloxacin-resistant isolates identified in this study (Table 2). Although found among clonally diverse strains (suggesting homoplasy), subtle amino acid substitutions such as Val130Ile (GyrB) and Leu406Ile (GyrA) would be unlikely to have a major impact on protein structure. In contrast, polymorphisms Ile139Arg (GyrB), Asp205Glu, Lys413Asn and Asp468Asn (GyrA) embody more dramatic amino acid substitutions. However, these were confined to related MLST groups and thus evidence for homoplasy and Darwinian selection at these sites is weaker. By comparison, the proven resistance-conferring mutation Thr82Ile in the GyrA QRDR is seen among diverse clonal frames (Fig. 1; Supplementary Fig. S1), consistent with an associated fitness advantage under antibiotic selection [5]. None the less, the current findings highlight the existence of diverse extra-QRDR mutations in C. difficile including polymorphisms within the GyrA dimer interface region such as Lys413Asn, Gly401Arg, Gly401Val and Gln418Lys.

Although alteration within the QRDR is clearly a dominant driver of fluoroquinolone resistance in $C$. difficile, the current findings demonstrate the capacity for mutational alteration outside this region to contribute to resistance. Given the confirmed ability of the GyrA Ala384Asp substitution to confer resistance and the numerous polymorphisms identified outside the QRDR of $C$. difficile DNA gyrase (in particular ca. residues 130-160 and 384468 of GyrB and GyrA, respectively), further investigation of extraQRDR substitutions and their association with fluoroquinolone resistance is warranted.

\section{Funding}

This study was wholly funded by the Department of Clinical Microbiology, Trinity College Dublin (Ireland).

\section{Competing interests}

None declared.

\section{Ethical approval}

Not required. 


\section{Acknowledgment}

The authors would like to thank the staff at St James's Hospital Microbiology Department (Dublin, Ireland) for their technical assistance and donation of isolates.

\section{Appendix A. Supplementary data}

Supplementary data associated with this article can be found, in the online version, at doi:10.1016/j.jgar.2015.09.007.

\section{References}

[1] Carroll KC, Bartlett JG. Biology of Clostridium difficile: implications for epidemiology and diagnosis. Annu Rev Microbiol 2011;65:501-21.

[2] Owens Jr RC, Donskey CJ, Gaynes RP, Loo VG, Muto CA. Antimicrobial-associated risk factors for Clostridium difficile infection. Clin Infect Dis 2008;46(Suppl. 1):S19-31.

[3] Johnson S, Samore MH, Farrow KA, Killgore GE, Tenover FC, Lyras D, et al. Epidemics of diarrhea caused by a clindamycin-resistant strain of Clostridium difficile in four hospitals. N Engl J Med 1999;341:1645-51.

[4] Drlica K, Hiasa H, Kerns R, Malik M, Mustaev A, Zhao X. Quinolones: action and resistance updated. Curr Top Med Chem 2009;9:981-98.

[5] He M, Miyajima F, Roberts P, Ellison L, Pickard DJ, Martin MJ, et al. Emergence and global spread of epidemic healthcare-associated Clostridium difficile. Nat Genet 2013;45:109-13.
[6] Dridi L, Tankovic J, Burghoffer B, Barbut F, Petit JC. gyrA and gyrB mutations are implicated in cross-resistance to ciprofloxacin and moxifloxacin in Clostridium difficile. Antimicrob Agents Chemother 2002;46:3418-21.

[7] Spigaglia P, Barbanti F, Louie T, Barbut F, Mastrantonio P. Molecular analysis of the gyrA and gyrB quinolone resistance-determining regions of fluoroquinolone-resistant Clostridium difficile mutants selected in vitro. Antimicrob Agents Chemother 2009;53:2463-8.

[8] Solomon K, Fanning S, McDermott S, Murray S, Scott L, Martin A, et al. PCR ribotype prevalence and molecular basis of macrolide-lincosamide-streptogramin $\mathrm{B}\left(\mathrm{MLS}_{\mathrm{B}}\right)$ and fluoroquinolone resistance in Irish clinical Clostridium difficile isolates. J Antimicrob Chemother 2011;66:1976-82.

[9] Stubbs SL, Brazier JS, O'Neill GL, Duerden BI. PCR targeted to the 16S-23S rRNA gene intergenic spacer region of Clostridium difficile and construction of a library consisting of 116 different PCR ribotypes. J Clin Microbiol 1999;37:461-3.

[10] Walsh P, Carroll J, Sleator RD. Accelerating in silico research with workflows: a lesson in simplicity. Comput Biol Med 2013;43:2028-35.

[11] Li H, Durbin R. Fast and accurate short read alignment with Burrows-Wheeler transform. Bioinformatics 2009;25:1754-60.

[12] Li H, Handsaker B, Wysoker A, Fennell T, Ruan J, Homer N, et al. The Sequence Alignment/Map format and SAMtools. Bioinformatics 2009;25:2078-9.

[13] Doran AG, Creevey CJ. SNPdat: easy and rapid annotation of results from de novo SNP discovery projects for model and non-model organisms. BMC Bioinformatics 2013;14:45

[14] Blanc-Potard AB, Labesse G, Figueroa-Bossi N, Bossi L. Mutation at the 'exit gate' of the Salmonella gyrase A subunit suppresses a defect in the gyrase B subunit. J Bacteriol 2005;187:6841-4.

[15] Devasia R, Blackman A, Eden S, Li H, Maruri F, Shintani A, et al. High proportion of fluoroquinolone-resistant Mycobacterium tuberculosis isolates with novel gyrase polymorphisms and a gyrA region associated with fluoroquinolone susceptibility. J Clin Microbiol 2012;50:1390-6. 